\title{
Gerhard Lampersbergs Dornröschen
}

\author{
Eine Rehabilitierung
}

Zum 100. Geburtstag von Robert Walser wurde erstmals ein Dramolett von Robert Walser vertont und 1978 am dritten und letzten Metamusik-Festival in Berlin uraufgeführt. Der österreichische Komponist und Schriftsteller Gerhard Lampersberg (1928-2002) verwandelte Walsers Dornröschen in eine Groteske im Stil der Wiener Gruppe um H. C. Artmann, Wolfgang Bauer und Konrad Bayer. Es war der erste Versuch, Robert Walser mit den künstlerischen Mitteln der Nachkriegsavantgarde neu zu interpretieren. Interessanterweise handelte es sich dabei nicht um eines der um die Jahrhundertwende geschriebenen Märchendramolette, die Walser 1919 unter dem Titel Komödie im CassirerVerlag herausgab, sondern um das im Folgejahr entstandene und publizierte Dornröschen.

Gerhard Lampersberg bildet in der Musikgeschichte den einmaligen Fall eines Komponisten, der durch einen Schlüsselroman wirkungsvoll aus dem Kreis anerkannter Künstler ausgeschlossen wurde. Wenn man Lampersbergs Namen erwähnt, fällt auch heute noch jener seines früheren Intimfreundes und späteren Erzfeindes Thomas Bernhard, der ihn in seinem 1984 publizierten Roman Holzfällen. Eine Erregung mit einer erbarmungslosen und rufmörderischen Hasstirade vernichtete. Zwar gab es durchaus Kritik an Bernhards Art, das in Österreich bekannte und mäzenatisch wirkende Ehepaar Lampersberg - im Roman behelfsmässig getarnt als »der Auersberger« und »die Auersbergerische« - auf menschlich bedenkliche Weise zu ruinieren, aber an der Berechtigung von Bernhards Kritik am »Snobund Geckmusikschreiber « ${ }^{1}$ wurde nicht gezweifelt und Lampersbergs Tätigkeiten als Komponist, Schriftsteller und kultureller Brückenbauer fortan nicht mehr ernst genommen. Niemand wagte es, Bernhards Einschätzung von Lampersberg anzuzweifeln, zumal Bernhard einst am Mozarteum Salzburg Regie studiert hatte und ein begabter Sänger gewesen war. Auch Bernhards

1 Bernhard: Holzfällen, S. 61. 
äußerst konservative musikalische Einstellung und seine Aversion gegen die Zwölftontechnik, die ihn veranlasste, Webern als »Schwächeanfall der Musikgeschichte« zu bezeichnen, wurde kaum kritisch reflektiert:

Wie Bruckner unerträglich monumental, so ist Webern unerträglich dürftig und noch hundertmal dürftiger als der dürftige Anton von Webern ist der Auersberger, den ich, wie die stumpfsinnigen Literaten den Paul Celan sozusagen als beinahe wortlosen Dichter, als beinahe tonlosen Komponisten bezeichnen muß. Der steiermärkische Epigone ist ja nicht unaufgeführt, denke ich, aber er ist schon vor dreißig Jahren, also schon in der Mitte der Fünfzigerjahre in der Webernnachfolge steckengeblieben; keine drei Töne sind von ihm, denke ich, aus dem nichts geworden ist. Den Kompositionen des Auersberger fehlt der Auersberger, denke ich, seine sogenannte aphoristische Musik (so meine eigene Bezeichnung für sein Kopieren als Komponieren in den Fünfzigerjahren!) ist nichts als ein unerträglicher epigonaler Webern, der ja selbst, wie ich jetzt weiß, kein Genie, nur ein plötzlicher, wenn auch genialer Schwächeanfall der Musikgeschichte gewesen ist. $^{2}$

Ilija Dürhammer hat in seiner umfassenden Studie unter anderem zu Bernhards Homosexualität den Spieß umgedreht und wenn auch nicht den Komponisten, so doch den kulturellen Brückenbauer Lampersberg rehabilitiert, indem er dessen Bedeutung für die literarische Entwicklung von Thomas Bernhard hervorhebt, der anfänglich noch »Bilder ländlicher Heimatidylle malte«. Erst durch das Ehepaar Maja und Gerhard Lampersberg wurde er »in eine intellektuelle Gesellschaft ersten Ranges eingeführt«; Lampersberg »wies [...] Bernhard den Weg in die österreichische Avantgarde «. ${ }^{3}$

1954 vermählten sich Gerhard Lampersberg und die Sängerin Maja WeisOstborn, die aus einer altösterreichischen Aristokratenfamilie stammte. $\mathrm{Zu}$ ihrer Mitgift zählte der sogenannte Tonhof, ein geräumiger Gutshof mit Scheune in Maria Saal bei Klagenfurt. Dorthin lud das Ehepaar Lampersberg während vier Jahrzehnten einen Großteil der literarisch-musikalischen Avantgarde Österreichs ein, zu Versammlungen, Konzerten, Theateraufführungen und Ausstellungen in der umgebauten Scheune, aber auch zu längeren Studienaufenthalten. Neben Thomas Bernhard fanden sich dort im Laufe der Jahre die Dichterin Christine Lavant, die Schriftsteller Gerhard Rühm, H. C. Artmann, Wolfgang Bauer, Peter Handke, Gert Jonke, Peter Turrini und Josef Winkler sowie die Komponisten Friedrich Cerha, Anestis Logothetis und Otto M. Zykan ein. ${ }^{4}$

2 Ebd., S. 6if.

3 Dürhammer: Geheime Botschaften, S. 358.

4 Maja Lampersberg gab auch künstlerische Vermittlungskurse für die Kinder von Maria Saal; diese >Tonhofkinder< durften malen und Musik machen. Später wurde der Begriff 
In zahlreichen Fällen kam es zur künstlerischen Zusammenarbeit zwischen Gerhard Lampersberg und den eingeladenen Schriftstellern. Diese war gerade im Falle von Thomas Bernhard besonders intensiv; Bernhard schrieb drei Libretti für Lampersberg. ${ }^{5}$ Dürhammer erkennt in Bernhards Tonhof-Zeit die entscheidende Wende in seinem Schaffen:

Die offensichtliche Heimatdichtung mußte Bernhard nun sein lassen, um sich in diesem Kreis nicht lächerlich zu machen. Die Avantgarde führte bei ihm erst zu einer Nachahmung und dann, als er sich auch sozial zurückzog, nach und nach zu einer gekonnten Synthese zwischen dem großväterlichen Erbe und der »modernen Form«, die aus der Auseinandersetzung mit der Avantgarde resultierte. ${ }^{6}$

Das Verhältnis zwischen dem Ehepaar Lampersberg und Bernhard war allerdings mehr als nur ein künstlerischer Austausch, denn die Bekanntschaft entwickelte sich zu einer Ménage-à-trois, die vor allem von der Liebe der beiden Männer zueinander dominiert war. Die Beziehung ist zwar in Form einer Erinnerung in Holzfällen (»jahrzehntelang peinigend abstoßend für mich «) erwähnt, ${ }^{7}$ Dürhammer macht aber deutlich, wie intensiv diese Liebe war, die zeitweilig zum Auszug von Maja Lampersberg führte. ${ }^{8}$ Das erklärt auch, weshalb der Bruch zwischen Lampersberg und Bernhard nach dem Sommer 1960, als im Tonhof die gemeinsame Oper köpfe unter der Leitung von Friedrich Cerha uraufgeführt wurde, so unerbittlich und radikal war. Denn der äußerliche Anlass für das Zerwürfnis waren eher harmlose Provokationen: Bernhard zog während der Probenzeit ohne Rücksprache mit Lampersberg ins beste Hotel Klagenfurts und ließ die Rechnung an den Tonhof schicken, und obwohl er bei den Proben anwesend war, bedauerte er nach der Uraufführung

>Tonhofkinder auf alle Besucher des Gutshofs ausgeweitet. Von der Bevölkerung in Maria Saal wurden die Kunstaktionen des Tonhofs, die auch in der freien Gegend veranstaltet wurden, oft als bizarr wahrgenommen und nicht verstanden; deshalb fand noch im Jahre 20oo die geplante Ernennung Lampersbergs zum Ehrenbürger im Gemeinderat von Maria Saal keine Mehrheit.

5 Vgl. Bernhard: Dramen I. Pia Janke erwähnt auch noch das Gartenspiel für den Besitzer eines Lusthauses in Kärnten, das ihr als Typoskript von Herbert Gamper zur Verfügung gestellt wurde. Dieses Libretto wurde in die Werkausgabe nicht aufgenommen. Vgl. Janke: Thomas Bernhard als Librettist, S. 217-227.

6 Dürhammer: Geheime Botschaften, S. 358.

7 Bernhard: Holzfällen, S. 167 .

8 Dürhammer: Geheime Botschaften, S. 426-432. Schon früher hat Alfred Pfibigan auf den homosexuellen Aspekt von Holzfällen hingewiesen, vgl. Pfibigan: Holzfällen. Männliche Homosexualität der Bernhardschen Prosa. 
in einem seiner berühmten Leserbriefe, dass er als Autor leider erst an der Premiere sein Stück habe sehen können. ${ }^{9}$

Dürhammers Nachweis der Ménage-à-trois mag erklären, weshalb Bernhard in Holzfällen gerade das Ehepaar Lampersberg mit solch beißendem Spott belegt und nur bei ihnen und nicht dem ganzen Figurenarsenal privateste und intimste Details preisgibt. Im Roman geht es nicht nur darum, die Wiener Kulturschickeria zu kritisieren, die alle »ganz einfach nicht das Höchste erreicht $«^{10}$ haben, sondern seine frühere Liebe zum Ehepaar Lampersberg definitiv und bleibend zu zerstören. Deshalb ist Bernhards Umgang mit den anderen Vertretern des Tonhof-Kreises, die in Holzfällen ebenfalls kaum verschleiert auftreten, deutlich milder. ${ }^{11}$ Sie verkörpern zwar »diese Art von epigonaler scheinintellektueller Geschwätzigkeitsliteratur«, aber die Kritik verbleibt hier im literarischen Bereich. ${ }^{12}$

Lampersberg wird ein solches Niveau der Kritik nicht gegönnt. Er wird als unappetitlicher, von der Trunksucht verblödeter, nach seiner Frau schlagender und mit seinem Gebiss herumfuchtelnder Widerling dargestellt. Und wenn es um Musikalisches geht, schwingt deutlich die Eifersucht auf die internationalen Erfolge des Komponisten mit, dessen Werke der große Tragöde ihrer Kürze und Belanglosigkeit wegen veräppelt:

Die Kompositionen des Auersberger sind ja nicht unaufgeführt, [...] alle Augenblicke wird von ihm etwas gesungen, geblasen, gezupft (dafür sorgt er schon!), alle Augenblicke etwas vom ihm geschlagen oder gestrichen, einmal in Basel, einmal in Zürich, einmal in London, einmal in Klagenfurt (dafür sorgt er schon!), da ein Duett, dort ein Terzett, da ein Vierminutenchor, da eine

9 Vgl. die Aussage von Maja Lampersberg in Fialik: Der Charismatiker, S. $108 \mathrm{f}$.

10 Bernhard: Holzfällen, S. 61.

11 Es sind dies die Männer Ernst Jandl (1925-200o) (als »dichtender Lebensgefährte« von Friederike Mayröcker) und Fritz Riedl (1923-2012) (als »der Tapisseriekünstler Fritz«), Claus Peymann (*1937, als »ein neuer Burgtheaterdirektor«) sowie die Frauen Jeannie Ebner (1918-2004) (als Dichterin Jeannie Billroth) und Friederike Mayröcker (*1924) (als Gymnasiallehrerin Anna Schreker).

12 Bernhard: Holzfällen, S. 162. Der Bruch mit dem Wiener Literatenkreis geht auf einen Konflikt aus dem Jahr 1959 zurück. In einem Selbstportrait unter der Rubrik Junge Köpfe geisselt Bernhard in der Monatszeitschrift Morgen »die jungen und zäh älter werdenden Literaten dieser Stadt, die, Epigonen von Natur aus, in den Kaffeehäusern bei lebendigem Leib vermodern« und bedauert, bisher keinen »lebenden deutschen Dichter der Weltliteratur« gefunden zu haben. Das führte zur scharfen und ironischen Entgegnung aus dem Café Hawelka: »Was den einzigen deutschen Dichter von Weltruf betrifft - der nicht zu finden sei-, so möchten wir Bernhard auf sich selbst weisen. Ach, wie das Gute doch nah liegt!« Gezeichnet wurde der Brief von Jeannie Ebner, H. C. Artmann, Gerald Bisinger, Elfriede Gerstl und Kurt Klingler. Zit. nach Bernhard:Journalistisches. Reden. Interviews, Bd. 1, S. 577 und 8o7. 
Zwölfminutenoper, dort eine Dreiminutenkantate, da eine Sekundenoper, dort ein Minutenlied, da eine Zwei-, dort eine Vierminutenarie [...]. Kaum ist er in der einen Stadt angekommen, denkt er schon an die andere, [...] unser vielgereister trippelnder Auersberger, unser rastloser Webern- und Grafenkopist, unser Snobund Geckmusikschreiber aus der Steiermark. ${ }^{13}$

Lampersbergs Dornröschen-Fassung hätte Bernhard in die Gruppe der >Zwölfminutenopern< eingeordnet, denn sie dauert nur etwa eine knappe halbe Stunde, und er hätte sie sicher als >fanatisch-modisch< bezeichnet und als sschein-intellektuellen< Theaterversuch in der Tradition der Wiener Gruppe vernichtet.

\section{$5 \cdot 2$}

\section{Dornröschen am Metamusik-Festival Berlin}

Tatsächlich ist die Produktion von Dornröschen am dritten und letzten Metamusik-Festival 1978 indirekt mit der Wiener Gruppe verbunden, denn Walter Bachauer vom RIAS Berlin, der Lampersberg den Kompositionsauftrag vermittelte und die Uraufführung programmierte, war in Graz aufgewachsen und hatte anfänglich in Wien studiert und dort die Wiener Gruppe kennengelernt. Bachauer (1942-1989) war mitverantwortlich dafür, dass Lampersberg gerade im Berlin der 6oer- und 7oer-Jahre regelmäßig aufgeführt wurde. Er war wie Lampersberg ein großer Webern-Verehrer und zählte zu den maßgeblichen Musikredaktoren des RIAS in West-Berlin. Mit seinem AvantgardeMagazin Montag 23 Uhr wurde er weit über Berlin hinaus bekannt, weil er zu den ersten zählte, die sich über alle stilistischen Grenzen hinwegsetzten und die zeitgenössische Avantgarde mit außereuropäischer Musik, amerikanischer Minimal Music und Rockmusik ohne Vorurteile und Rücksichtsnahmen mischten und miteinander konfrontierten. Gerade für das Sendegebiet der DDR spielte Bachauer eine bedeutende Rolle. Vor seinen Sendungen - so Peter-Michael Hamel in seinem Nachruf - sollen leere Tonbänder in Ostberlin jeweils ausverkauft gewesen sein. ${ }^{14}$

\footnotetext{
13 Bernhard: Holzfällen, S. 61. Die Auswechslung von Kärnten durch die Steiermark zählt zu den behelfsmäßigen Tarnungen Bernhards.

14 Walter Bachauer begann 1964 beim RIAS als freier Mitarbeiter und wurde 1970 fester Redaktor. 1980 ernannte man ihn zum Hauptabteilungsleiter Musik, eine Stellung, die er bald verließ, um in Hollywood mit Francis Ford Coppola und Philip Glass bei der Filmmusik zu Koyaanisqatsi mitzuarbeiten. Walter Bachauer trat unter dem Pseudonym Clara Mondshine auch als Komponist elektronisch-psychedelischer Musik in Erscheinung und publizierte etliche Alben, in denen orientalisch-afrikanische Elemente mit Minimal
} 
Von 1974 bis 1978 leitete Bachauer vom RIAS aus mit verschiedenen institutionellen Partnerschaften das von ihm gegründete, zweijährlich stattfindende Metamusik-Festival Berlin. Gruppen aus der ganzen Welt wurden eingeladen und oft Gegensätzliches kombiniert. Das Festival wurde mit einfachen Mitteln und ohne jeden Schnickschnack durchgeführt. Die Programme zum Beispiel wurden mit Schreibmaschine abgetippt und dann hektografiert.

DerTitel des dritten und letzten Metamusik-Festivals lautete Musik \& Aktion und das Programmblatt der Zweitaufführung von Lampersbergs Dornröschen ist beispielhaft für Bachauers Programmierung (vgl. Abb. 15): Auf das Melodram Stallo (1961) - eine Zusammenarbeit Lampersbergs mit H. C. Artmann, die ein Jahr nach dem Bruch mit Thomas Bernhard entstanden war - folgte die Uraufführung von Dornröschen, und im Nachtkonzert konnte man einer buddhistischen Zeremonie von Mönchen des Klosters Enryaku-ji auf dem Berg Hieizan bei Kyoto beiwohnen. ${ }^{15}$

Die Besetzung für den Abend war hochkarätig, weil Lampersberg und Bachauer auf die Kräfte der Deutschen Oper Berlin zurückgreifen konnten, deren damaliger Intendant Siegfried Palm selber ein großer Förderer und als Cellist auch Interpret zeitgenössischer Musik war. Das Gesangsensemble war hervorragend besetzt, Caspar Richter ein auf zeitgenössische Musik spezialisierter Dirigent und Winfried Bauernfeind gewissermaßen der Hausregisseur der Deutschen Oper. Ein besonderer Glücksfall war der schon damals international bekannte neorealistische Maler Johannes Grützke, Mitbegründer der "Schule der neuen Prächtigkeit«, der die Ausstattung und die Kostüme verantwortete und in Nebenrollen auf der Bühne mitspielte. Die Aufnahme der Oper wurde im Rahmen des Programmaustauschs von Belgien, der Westschweiz, Griechenland, Portugal, den USA, Algerien, Australien, Malawi, Rumänien und Dänemark bestellt und mit hoher Wahrscheinlichkeit auch ausgestrahlt. ${ }^{16}$ Für Lampersberg war es eine der repräsentativsten Aufführungen seines Lebens. Robert Walser hatte als Autor nie zuvor eine solche internationale Ausstrahlung erfahren.

Music und Rock gemischt werden. Er schied am 16. Februar 1989 freiwillig aus dem Leben. Vgl. Hamel: ... auf Reisen ... Nachruf auf Walter Bachauer.

15 [o. A.]: Programm des Metamusik-Festival 3 vom 27.10.1978.

16 In der Sammlung Lampersberg im Kärntner Literaturarchiv befindet sich eine entsprechende Liste. Ob all diese Sender die Aufnahme ausgestrahlt haben, lässt sich nicht mehr verifizieren. Ausgehend von meinen eigenen Erfahrungen als Musikredaktor beim Schweizer Radio in den 8oer-Jahren waren im Programmaustausch bestellte Aufnahmen in aller Regel schon fest programmiert und wurden deshalb auch ausgestrahlt. 


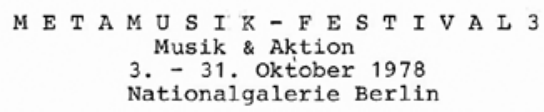

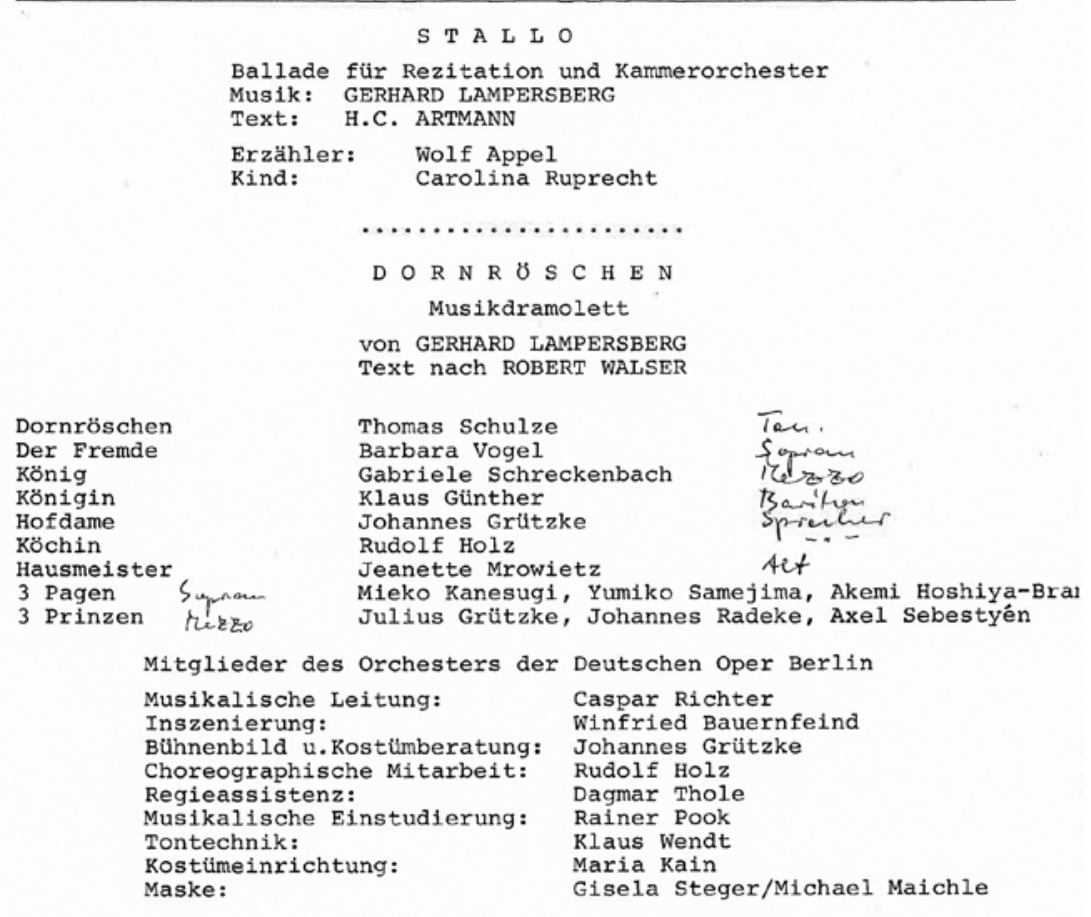

(Wiederholung vom 25. Oktober)

$$
\begin{array}{r}
\mathrm{SH} \hat{\mathrm{O}}-\mathrm{M} \mathrm{Y} \hat{\mathrm{O}} \\
\text { Buddhistisches Ritual }
\end{array}
$$

MÖNCHE DER TENDAI SEKTE VON HIEIZAN ENRYAKU-JI

(Wiederholung vom 26.Oktober)

Abb. 15 Programm des Metamusik-Festival vom 27.10.1978, S. 1 


\subsection{Zum Libretto}

Ähnlich wie Wladimir Vogel beim Dramma-Oratorio Flucht geht Gerhard Lampersberg mit Robert Walsers Text in einer fast übergriffigen Art um, die heute kaum mehr akzeptiert würde. Wie er sich ein ideales Libretto vorstellte, erklärte Lampersberg 1991 am Beispiel von Thomas Bernhard im Gespräch mit Maria Fialik folgendermaßen:

Nun muß ich sagen, ich habe den Text ja nicht so verwenden können, wie er [Bernhard] ihn gemacht hat. Ich habe ihm ein Stück von [H. C.] Artmann gezeigt, der knabe mit dem brokat. ${ }^{17}$ Und ich habe dem Bernhard gesagt: »Das ist das Muster, ein ideales Libretto für meine Musik, also knapp, ein Skelett brauche ich. Dazwischen muß alles liegen, geheimnisvoll und nicht direkt gesagt. «18

In diesem Sinne hat Lampersberg Walsers Text denn auch >skelettiert<, das Stück auf die dramaturgische Grundstruktur reduziert und das kurze Dramolett auf ein knappes Drittel gekürzt. Damit schaffte er sich Freiräume für die eigene Musik. Die allgemeine Aussage des Werks beschreibt Lampersberg im Vorwort zum Libretto folgendermaßen:

Walsers Dornröschen wandelt scherzhaft noch einmal das Thema von Aschenbrödel und Schneewittchen ab, den nie zu schlichtenden Konflikt von Kunst und Leben: Schönheit und Liebe sind real nur im Märchen, im Traum und [in der] Phantasie, der Träumer ist einsam unter den Menschen, ihnen ein Fremder und fremd sie ihm.

Walsers kaum charakterisierte Personen erhalten in Lampersbergs Auffassung ein klares Profil:

Dornröschen als Sehnsuchtsjungfer, der Prinz als Antiheld, das Königspaar in leicht einfältiger Würde, die Hofdamen penetrante, lüsterne Gouvernanten, der Hofdichter ein schwärmerischer, infantiler Pagentyp, der Hausmeister gutmütig polternd, der Koch selbstbewußt-energisch, die Köchin beschränkt-ergeben, der Jäger ein charmanter Luftikus, der Diener ein dümmlicher Tollpatsch, die Mamsell ein kokett-geziertes Fräulein.

17 Artmann/Lampersberg/Vennekamp: Der Knabe mit dem Brokat. Die Oper wurde zwischen 1954 und 1963 dreimal vertont und alle Versionen sollten bei einer Aufführung nacheinander gespielt werden. Vgl. Fialik: Der Charismatiker, S. 217 (Anm. 17).

18 Ebd., S. 59 . 
Zu Walsers Sprache meint Lampersberg:»[D]ie Poesie ist absichtlich überdeckt von banalem hausbackenem Gerede. ${ }^{19}$ Dieses >Geredeく strich Lampersberg im Libretto großenteils weg; er stellte eine Prosafassung her, in der Walsers Versstruktur weitgehend aufgelöst ist. Damit fallen auch viele poetischkunstvolle Umständlichkeiten weg, die dem Versrhythmus geschuldet sind; zudem beseitigt Lampersberg alle selbstreflexiven Momente, wie die prompte Antwort des anfänglich verstockt-stummen Fremden auf die Frage, ob er denn nicht reden könne: »Ich rede nachher noch genug, / es wird wohl nicht so schrecklich eilen.« (SW 14, 168)

Aus dieser Kürzung entsteht ein krud-trockenes Libretto, das an Operettentexte erinnert, viele Wechsel der Stimmen aufweist und MonologischReflektierendes ausschließt. Vor allem führt Lampersberg neu einen allwissenden kommentierenden Sprechchor ein, der in der Tradition von Vogels Sprechchören in metrisierter Rhythmik spricht, allerdings immer monophon und rhythmisch weniger ausgefeilt. Das Libretto, das bei der Uraufführung auf zwei A4-Seiten Platz fand, erinnert an jene Texte, die Artmann und Bernhard für Lampersberg im Stile der Wiener Gruppe geschrieben haben: Kurze Sätze, Wortrepetitionen, Imitation von gleichen oder ähnlichen Formulierungen durch andere Personen oder durch den neu eingeführten Chor. Auch wenn man literarisch betrachtet die vielen Striche bedauern mag, hält Lampersberg alle Fäden des ursprünglichen Textes zusammen und schafft, von einem dramaturgischen Standpunkt aus, ein theaterwirksames Libretto.

Wahrscheinlich ist diese angestrebte Theaterwirksamkeit auch der Grund, weshalb sich Lampersberg für Dornröschen entschieden hat, das schon von Walser selbst viel holzschnittartiger gefasst wurde als die in Selbstreflexionen kreisenden frühen Dramolette Schneewittchen und Aschenbrödel. Die zentralen Elemente der Dornröschen-Handlung bilden einerseits der mühelos durch die Dornenhecken spazierende Fremde, dem wegen des mangelnden Abenteuers der Helden-Status fehlt, und andererseits der von ihm aufgeweckte Hofstaat, der lieber weitergeschlafen hätte. Dornröschen hätte gerne einen wackeren

19 Lampersberg: Einführender Kommentar zum Klavierauszug von Dornröschen. Dieser ausführliche Kommentar wurde in die eigentlichen Aufführungsunterlagen des Metamusik-Festivals nicht übernommen bzw. sehr stark redigiert. Die Mappe 382/W1-G in der Österreichischen Nationalbibliothek umfasst insgesamt 148 Seiten mit dem Entwurf eines Klavierauszugs, dessen Reinschrift mit Korrepetitionskommentaren, den Orchesterstimmen von Harmonium, Klavier und Celesta, die vom ORF auf Band aufgenommen wurden, und vom Schlagzeug, aber keine Partitur. Der Autor hatte noch in den 199oer-Jahren die Partitur bei der Universal Edition ausgeliehen. Der Verlag stellte aber inzwischen den Vertrieb der Oper ein und die Partitur liegt im Archiv der UE nicht mehr vor. 
Helden gehabt und nicht einen, wie »Der Fremde« - so heißt der Prinz bei Walser -, dem alles ohne Anstrengung in den Schoß fällt.

Dieses Handlungsmuster erinnert durchaus an slapstickartige Umschaltungen in der Tradition komischer Opern, etwa Orphée aux Enfers von Jacques Offenbach, in der die Beziehung zwischen dem eitlen Geigenlehrer Orpheus und seiner vor ihm fliehenden Eurydike so zerrüttet ist, dass Orpheus nach ihrem Tod hocherfreut ist und sich nur widerwillig und unter dem Druck der Gestalt der `Öffentlichen Meinung` auf den Weg macht, sie aus der Hölle $\mathrm{zu}$ befreien. In gewissen Sequenzen erinnert Walser direkt an Operettenlibretti, bei denen die Dialoge zwischen den verschiedenen Figuren wie Zahnräder ineinandergreifen: »es ginge ja nicht anders. / [...] weil's ohne mich gewiß nicht ginge. / Nein, es ginge nicht. / So aber geht's; / ja, ja, so geht es.« (SW 14, 175f.)

Solche Passagen hat Lampersberg belassen, darüber hinaus strich er die Vorlage weiter zusammen, um zusätzliche solche ineinandergreifende Dialogszenen zu erhalten.

Am Beispiel des Schlusses, bei dem Lampersberg eher wenig eingreift, lässt sich seine Bearbeitungsweise gut beobachten. Die linke Spalte zeigt Walsers Original; fett gesetzte Stellen markieren, was Lampersberg ins Libretto übernimmt; die kursiv gesetzte Stelle verschiebt Lampersberg und bildet mit ihr den neuen Schluss der Oper. In der rechten Spalte sind in eckigen Klammern die sprachlichen Eingriffe von Lampersberg vermerkt.

\section{Königin:}

Willst du den Schritt nicht überlegen?

Bedenke, was du da versprichst.

Dornröschen:

Würd' ich mir's lang noch überlegen, so könnt' es mir am End' verleiden.

Nein, ich bin mit mir selbst durchaus einig, und er ist jetzt mein Herr, zwar hätt' ich mir den Helden anders denken mögen, viel hübscher, etwas gefälliger und eleganter,

hinreiß'nder auch und in gewissem Sinne stolzer, ach, ich kann es nicht sagen, muß ihn nun eben nehmen, wie er ist, und tu's auch herzlich gern.

Der Fremde:

Dein allezeit galanter Diener! Und sollt' ich dir nur halb gefallen, du dich beinahe zwingen müßtest, [du beinah dich zwingen müßtest] 
mich anzusehen und zu lieben und dulden, nun, so sag' ich dir, was ein französisch Sprichwort sagt:

L'appétit vient en mangeant. Hoffe, [hoff ich, ] daß mir's gelingt, dich zu befried'gen. [daß es mirgelingt, dich zu befried'gen] Dornröschen:

So sei es! Macht Musik und laßt uns alle zusammen fröhlich sein. Die Sonne leuchtet und der Himmel ist bläulich, und die Winde fächeln uns unbefangen Kühlung zu.

Dies Schloß ist nun lebendig, alle woll'n wir uns künftig munter trauen und eifrig helfen, wo es not tut, uns heiter in die Augen blicken, vergnüglich miteinander leben und solchermaßen all's in allem gedeihliche Gesellschaft bilden.

König:

Da sprichst du gar nicht übel, Kind, ich bin dabei,

Königin:

$$
\text { ich auch, }
$$

Der Fremde: $\quad$ und ich auch, denn es ginge ja nicht anders. Dornröschen:

Ich auch, weil's ohne mich gewiß nicht ginge.

Der Fremde:

Dornröschen:

Nein, es ginge nicht.

So aber geht's;

Der Fremde:

Dornröschen:

ja, ja, so geht es.

Reden wir länger, so wird uns die Suppe kalt, drum brechen wir ab und geh'n jetzt vereint zu Tisch. Darf ich um deinen Arm dich bitten? [ich bitte dich um deinen Arm] Alle:

So wär' die Sache angenehm beendet mit 'ner frohen Hochzeit. (SW 14, S. 174-176)

[So reden wir nicht länger,] [sonst wird die Suppe kalt, ]

[Chor: Die Sonne leuchtet, blau ist der Himmel, die Winde fächeln kühl. Dies Schloss ist nun lebendig.] 
Lampersbergs neuer Schluss zeigt deutlich die >Operettisierung` von Walsers Text. Der ursprüngliche, sanft ironische Schluss, der »die Sache angenehm / beendet«, taugt nicht für eine gute Finalwirkung. Deshalb schneidet Lampersberg eine Passage aus Dornröschens Part aus und setzt sie an das Ende; auch hier wird Walsers Sprache vereinfacht: Der Himmel ist nicht »bläulich«, sondern »blau«, und die »unbefangen Kühlung« zufächelnden Winde dürfen bei Lampersberg nur noch kühl fächeln.

Durch die Striche fallen die von Walser ausgestalteten delikaten Momente weg, etwa Dornröschens ausführliche Beschreibung ihres Traummannes oder des Fremden Rückgriff auf ein französisches Sprichwort. Manchmal wird durch Lampersbergs Eingriffe die Bedeutung einer Wendung ins Gegenteil gekehrt, etwa wenn der König bei Walser nach einer ihn wegen ihrer Ausführlichkeit und gedanklichen Abgeklärtheit überraschenden Rede der Tochter sagt »Da sprichst du gar nicht übel « (SW 14, 175), während sich diese gleiche Bemerkung bei Lampersberg auf Dornröschens kurzes Fazit »Macht Musik und laßt uns fröhlich sein« bezieht.

So entsteht ein robustes, dramaturgisch wirkungsvolles Libretto, dem zwar die Magie der Walser'schen Sprache abhandengekommen ist, das aber für das Aktionstheater, das Lampersberg im Sinn hatte, bestens geeignet ist.

\subsection{Operetten-Aktion}

In der Einführung, die am Metamusik-Festival dem Programmzettel beigelegt war, schreibt Lampersberg:

Dornröschen ist ein bukolisches Verkleidungsspiel ohne mutwillige Absicht oder Verzerrungstendenz und ganz ohne psychologisierende Hintergedanken. Eine tiefere Bedeutung in dieser Travestie suche der Betrachter selbst. ${ }^{20}$

Das ist bewusst vorsichtig formuliert, denn bei der musikszenischen Umsetzung von Dornröschen setzt Lampersberg von Anfang an aufs groteske Register. Er wählt als drastisches Mittel die Travestie sämtlicher Rollen: Männerrollen werden von als Männer verkleideten Frauen gesungen und Frauenrollen von als Frauen verkleideten Männern. ${ }^{21}$ Dabei wird in der

\footnotetext{
20 [o. A.]: Programm des Metamusik-Festival 3 vom 27.10.1978.

21 Solche Verkleidungen haben Lampersberg auch noch im Alter interessiert. Am Tonhof-Symposion an der Sankt Veiter Literaturwoche 1991 wurde der Film Lendliche Langeweile - eine Tonhof-Travestie von Hermann Jamek mit Wolfgang Bauer, Ernst Kölz,
} 
Stimmlage keinerlei Imitation der Travestie gesucht: der Königin-Bariton singt nicht wie eine Frau (zum Beispiel unter Verwendung der Kopfstimme), und die König-Sopranistin ahmt keine Männerstimme im tiefen Stimmregister nach. Auf diese Weise bleibt die Travestie als Verkleidung immer präsent. Das unterscheidet Lampersbergs Vorgehen von den zahlreichen Hosen- bzw. Rockrollen der Operngeschichte. Zum Wesen der Hosenrolle gehört gerade, dass die Travestie gelingt und das Publikum der Illusion verfällt, es handle sich bei der Schauspielerin um einen - oft androgynen oder unreifen - Mann. Beim Dornröschen wäre das allenfalls bei der Figur des Fremden gelungen, denn dessen Kostüm orientierte sich in der Uraufführung am berühmten Bild, das Karl Walser von seinem adoleszenten und nach Friedrich Schillers Die Räuber als Karl Moor verkleideten Bruder Robert gemalt hatte. In der musikalischen Walser-Rezeption ist die Feminisierung des lyrischen Ichs in den Walser-Texten fast schon zur Tradition geworden. Allerdings konnte sich bei Lampersbergs Dornröschen dieser androgyne Zauber gerade nicht entfalten. Lampersberg lässt Dornröschen von einem überspannt-burschikosen Tenor darstellen, etwa wenn er bei der gesprochenen Textstelle »und von dem Kusse wacht ich auf« die Anweisung gibt: »frei, geschrien«. Die Szenenfotos, die von der Uraufführung erhalten sind, zeigen eine Maskerade: aufgeklebte Schnurrbärte, extravagante Perücken, viel Schminke und im Spiel selbst vermutlich Anleihen beim Schmierentheater.

Das Stück wird umrahmt von zwei Instrumentalstücken als Vorspiel und Finale, beide vergleichsweise lang. Das kleine Orchester besteht aus zwölf Musikern: drei Holzbläser (Flöte/Piccolo/Altflöte, Klarinette/Es-Klarinette und Fagott/Kontrafagott), drei Blechbläser (Trompete, Horn und Posaune), zwei Schlagzeuger und vier Streicher (Violine, Viola, Violoncello und Kontrabass). Dazu kommen die drei Tasteninstrumente Klavier, Harmonium und Celesta, die eine `Sphärenmusik« spielen, deren Part Lampersberg - wohl aus praktischen Gründen ${ }^{22}$ - im Österreichischen Rundfunk (ORF) vorproduziert hatte und während der Aufführung ab fünf verschiedenen RevoxTonbandgeräten einspielte.

Maja und Gerhard Lampersberg sowie Heidi Skorianz präsentiert, in dem Lampersberg und Bauer beide geschminkt und in Frauenkleidern auftreten. Lampersberg spielt Klavier und Bauer, der auf dem Nachttopf sitzt, ruft zum Schluss der Aktion aus: »Das ist meine Literatur!« Vgl. Fialik: Der Charismatiker, S. 67f. und 216 (Anm. 22).

22 Drei Tasteninstrumente in die Nationalgalerie zu stellen und aufeinander abzustimmen sowie zusätzlich drei Tastenspieler zu finden, dürfte die Möglichkeiten des Raumes und des Festivals überschritten haben. 
Die sehr tiefen und sehr hohen Instrumente (Kontrabass und Kontrafagott bzw. Trompete und Piccolo) dominieren, was auf das Schrille und Groteske auch in der musikalischen Umsetzung verweist. Den wichtigsten Part spielen die beiden Schlagzeuger, die neben den Pauken vor allem sunkonventionelle< Instrumente spielen: Gong, Tamtam, Pauke, kleine Trommel, Bongos, Triangel und Kastagnetten.

12
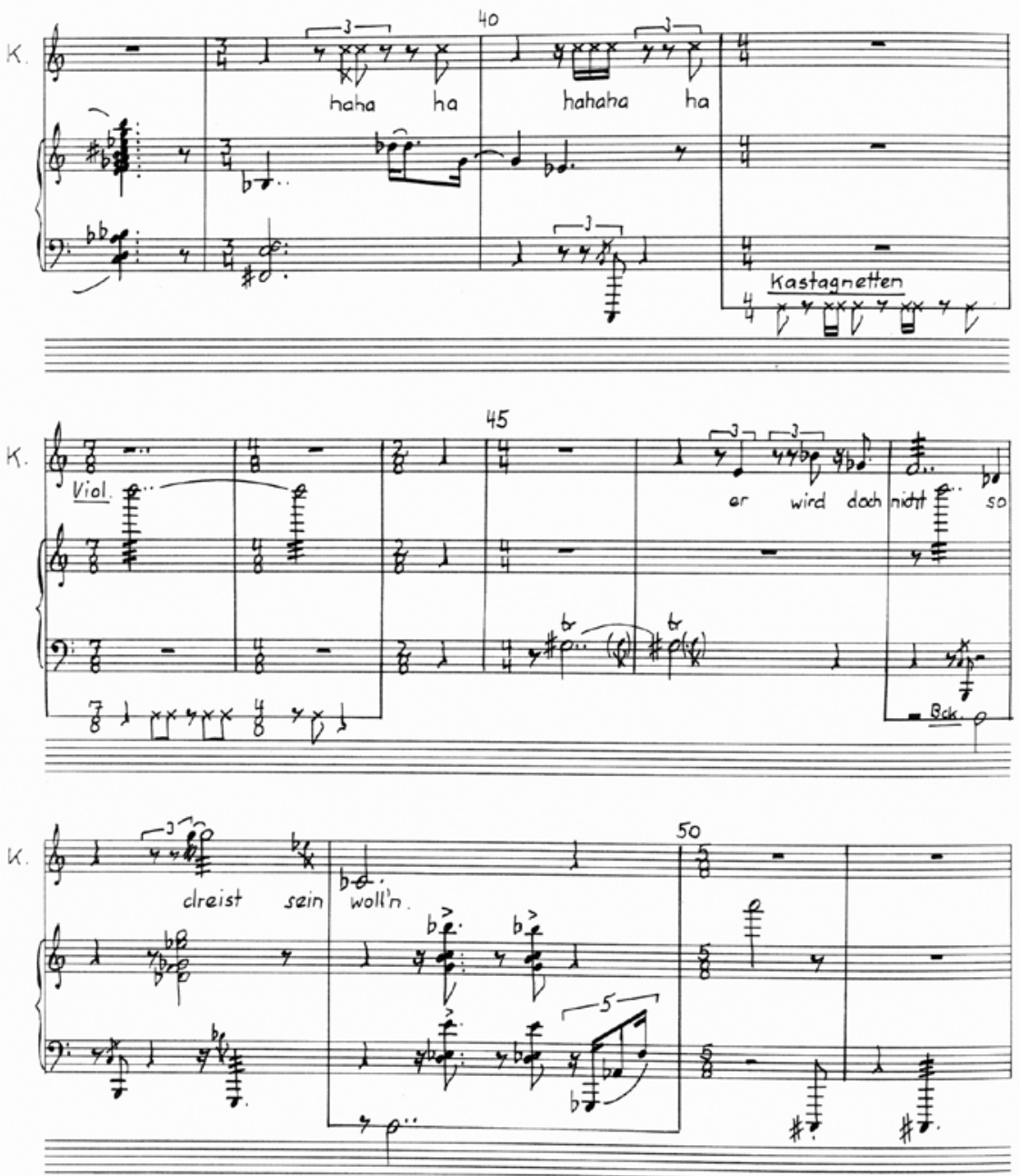

Abb. 16 Gerhard Lampersberg: Dornröschen, Klavierauszug, S. 12, Takte 38-51 
Gerade die Kastagnetten verwendet Lampersberg wie ein Leitmotiv, das er in der Oper in Kombination mit dem mächtig ausgebauten »Haha« des Königs einsetzt (vgl. Abb. 16) ${ }^{23}$ und das später von den Gesangsstimmen imitiert wird. Diese Schlaginstrumente sind insofern >auffällig $<$, als sie sich kaum mit anderen Instrumenten mischen und signalartig auf sich selbst verweisen. Während sich ein Geigenton in den musikalischen Kontext integriert, bleibt eine Kastagnette meist eine Kastagnette mit ihren spanischen Konnotationen, und zwar ganz unabhängig davon, was sie konkret spielt. Ähnlich verhält es sich mit dem Tamtam und dem Gong. Dieses dissoziative Verfahren, bei dem die Elemente nicht im herkömmlichen Sinne komponiert und zusammengeführt werden, sondern wie ein Mobile ineinandergreifen, ist das Grundprinzip von Lampersbergs Vertonung. Dieses Prinzip zeigt sich auch in der oben besprochenen Bearbeitung des Librettos, das nicht linear abläuft, sondern einzelne Sätze aus dem Kontext reißt und wiederholt. So besteht die Partie der Königin bis knapp vor Schluss nur aus dem Satz: »Gewiss, er hat das Schloss befreit und uns erlöst aus Zauberbann«, der fünfmal in fünf Varianten und fünf Konstellationen auftaucht. Lampersberg lässt in seiner Mobile-Technik die Stimmen einander überlagern, mischt Sprechstimmen und Singstimmen. Wegen der zahlreichen Wiederholungen wird der Text trotz der Überlagerungen am Ende verstanden.

Bei der Königin lässt sich auch Lampersbergs Verwendung der Zwölftontechnik gut aufzeigen. Ausgerechnet der »in der Webernnachfolge steckengeblieben[e] « Komponist, wie es bei Thomas Bernhard heißt, ${ }^{24}$ geht sehr frei mitder Dodekaphonie um. Erverzichtet auf eine Grundreihe, die alle Tonhöhen strukturiert, was für Webern undenkbar gewesen wäre. Vielmehr komponiert er verschiedenste Zwölftonreihen - im Sinne von Zwölftonkomplexen -, die einander mehr oder weniger organisch folgen. In reiner Form zeigt sich dies beim zweiten Einsatz der Königin (vgl. Abb. 16). Alle Varianten des Satzes behandelt Lampersberg gleich: Er bringt bei jedem gesungenen Halbsatz acht verschiedene Tonhöhen, die dann in der instrumentalen Begleitung zur Zwölftönigkeit ergänzt werden, sei es mit Akkorden, einem Nachspiel oder - wie hier - mit der Oboenmelodie. Statt mit einer fixen Reihe arbeitet Lampersberg meist mit Viertongruppen, die er ineinanderfügt. So bilden die Singstimme und die Oboe einen Zwölftonkomplex.

23 In seiner rhythmischen Präzision und Schärfe ist es wohl eine Anspielung an die 1. Szene von Alban Bergs Wozzeck mit dem mechanisch-sarkastischen Gelächter des Hauptmanns über Wozzecks Ungebildetheit.

24 Bernhard: Holzfällen, S. 61. 


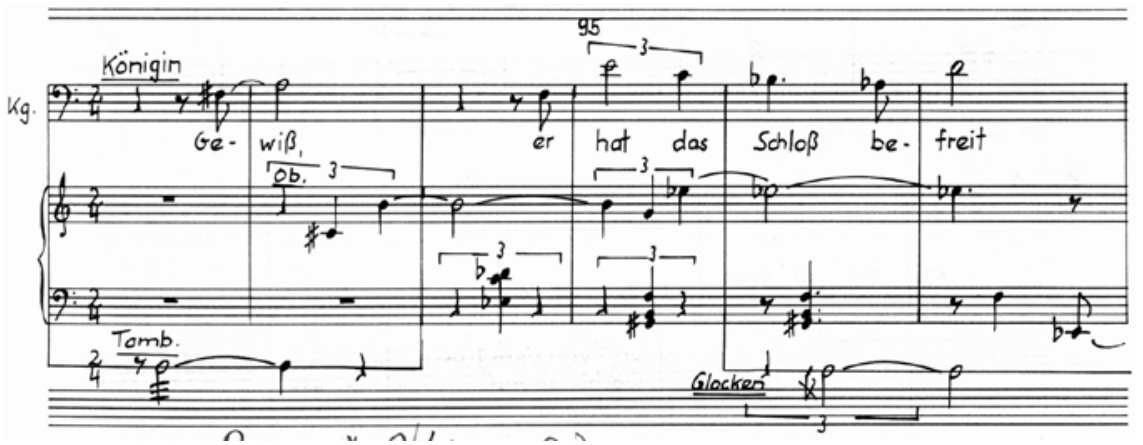

Abb. 17 Gerhard Lampersberg: Dornröschen, Klavierauszug, S. 15, Takte 92-97

Das Beispiel weist folgende Viertongruppen auf:

$\begin{array}{llll}\text { fis } & a & f & e^{1} \\ \text { Ge- } & \text { wiß } & \text { er } & \text { hat } \\ c^{1} & b & \text { as } & d^{1} \\ \text { das } & \text { Schloß } & \text { be- } & \text { freit } \\ \text { cis } & h^{1} & g^{1} & e s^{2} \\ \text { [Oboenmelodie] }\end{array}$

Wenn man diese Tongruppen in einer Oktave zusammenzieht, um die dodekaphone Struktur zu verdeutlichen, ergeben sich oft `Tetrachorde< vom Umfang einer reinen oder übermäßigen Quarte, deren Binnenstruktur dann unterschiedlich gestaltet wird. ${ }^{25}$ Im Falle des obigen Beispiels ergeben sich folgende Vierton-Gruppen:

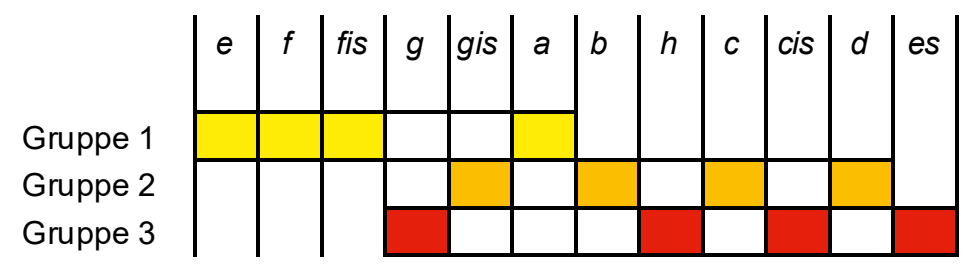

Abb. 18 Gerhard Lampersberg: Dornröschen, Struktur der Tetrachorde,

T. $92-97$

25 Derartige nicht-diatonische $>$ Tetrachorde $\prec$ sind auch für Heinz Holligers Komponieren im vollchromatischen Raum typisch (vgl. Kap. 10). 
Die erste Viertongruppe hat eine Quarte Umfang und in der Binnenstruktur dominieren kleine Sekunden, die zweite Gruppe besteht ausschließlich aus Ganztönen und verdeutlicht musikalisch das befreite Schloss; die letzte Gruppe setzt sich aus den vier noch fehlenden Tönen des Zwölftonfeldes zusammen.

Die fünfte und letzte Wiederholung des Satzes der Königin zeigt, wie sich in diesem mobile-artigen Komponieren die Prozesse verdichten können und der Dialog in seiner linearen Logik aufgelöst wird (vgl. Abb. 20). Wenn man die von der Königin gesungenen Töne wiederum in einer Oktave zusammenzieht, resultieren erneut zwei Tetrachorde; beide haben den Umfang eines Tritonus und stehen in einem spiegelsymmetrischen Verhältnis zueinander:

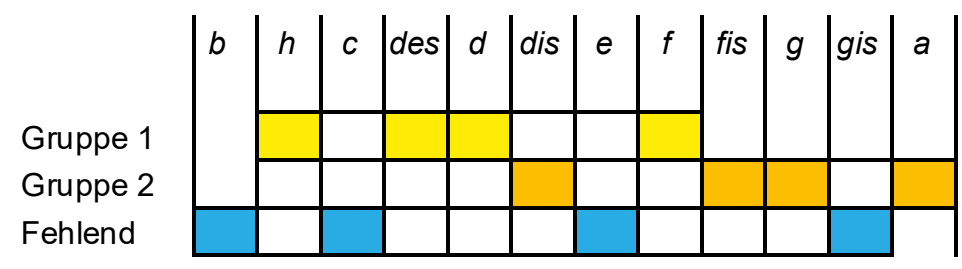

Abb. 19 Gerhard Lampersberg: Dornröschen, Tonhöhenschema, T. 355-357

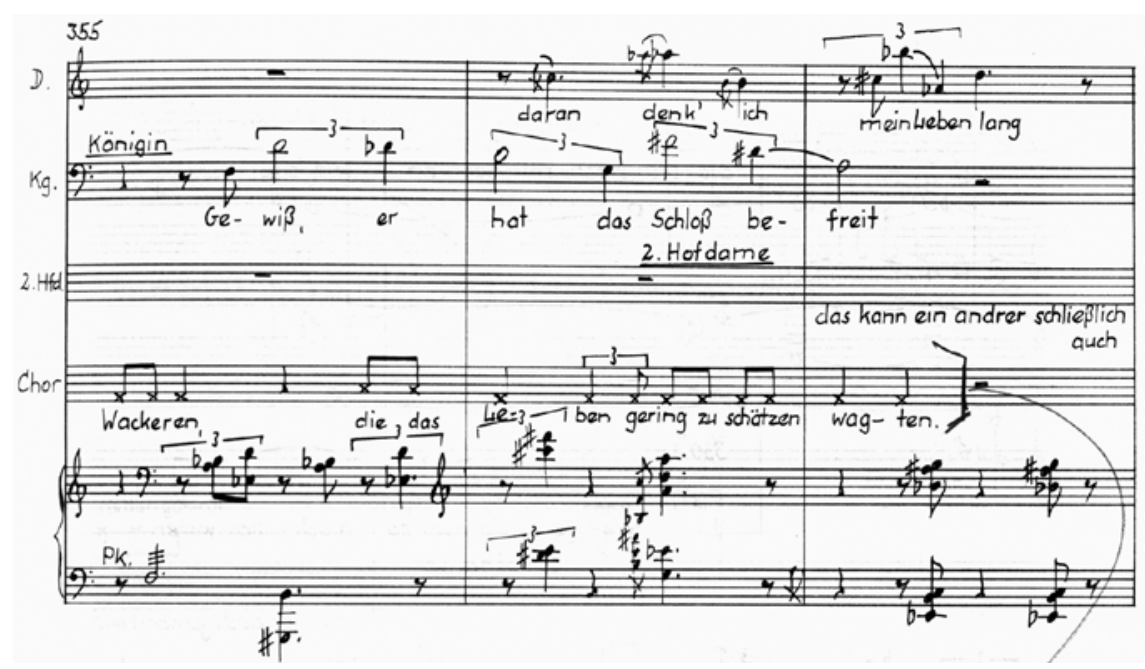

Abb. 20 Gerhard Lampersberg: Dornröschen, Klavierauszug, S. 35, Takte 355-357 
Um eine vollständige Zwölftonreihe zu erhalten, müssten die vier fehlenden Töne $c, e$, as und $b$ ergänzt werden. Und tatsächlich folgen diese Töne in der Partie von Dornröschen ${ }^{26}$ - mit $e^{1}$ und $c^{1}$ bei »daran« sowie $a s^{1}$ bei »denk'«; danach unterläuft Lampersberg ein $>$ Fehler<, weil beim »ich« von Dornröschen ein $h$ statt ein $b$ notiert ist, das aus der Systematik eigentlich folgen würde. Hat Lampersberg das Vorzeichen vergessen? Oder zog er die Terz $h-\operatorname{dis}^{1}$ der klingenden Quarte $b-$ dis $^{1}$ vor? Da die Wiederholung des $h$ in gleicher Oktavlage und im gleichen Takt ein klarer Regelverstoß gegen die Regeln der Zwölftonmusik darstellt, handelt es sich wohl tatsächlich um ein Versehen des Komponisten. Das Beispiel zeigt jedenfalls, dass Lampersberg sich bei Tonhöhenproblemen nicht zu lange den Kopf zerbrach und einmal Komponiertes nicht streng überprüfte. Viel wichtiger als die Nachkontrolle solcher Zwölftonbuchhaltungen sind die Sinn- und Stilüberlagerungen, die an dieser Stelle entstehen: Der rhythmisch sprechende Chor übernimmt die Rolle des allwissenden Kommentators und erinnert an die »Wackeren« (SW 14, 173) und `wahren Helden<, die in der Dornenhecke, im Versuch Dornröschen zu retten, ihr Leben ließen.

Dazu wiederholt die Königin in einer langgezogenen Kantilene zum fünften Mal ihren bisher einzigen Satz. Die zweite Hofdame wertet - gesprochen die Aussage der Königin ab, während Dornröschens Melodie bei »daran denk' ich mein Leben lang « in den Sprüngen und mit schleifenden Vorschlägen hysterisch-überspannt wirkt. Bei Walser bezieht sich Dornröschens Aussage deutlich auf die tapferen Helden, in Lampersbergs Vertonung bleibt der Bezug ambivalent und könnte sich wegen den Überlagerungen mit den anderen Stimmen auch auf den $>$ Fremden $<$ beziehen. ${ }^{27}$

Ähnlich wie den Anfang gestaltet Lampersberg auch das Ende der Oper als ein groteskes Ritual. Die Schlusspassage, die Lampersberg aus Material von Dornröschens Text neu zusammenstellt, wird vom Chor ohne Instrumentalbegleitung gesprochen. Dann folgt - gemäß Regieanweisung »schreitend, torkelnd« das Finale, bei dem die Schauspiel- und Sängertruppe abzieht. ${ }^{28}$

26 Die Partie des Dornröschen ist eine Oktave tiefer zu lesen, da sie für Tenorstimme komponiert ist.

27 In diesem Satz von Dornröschen könnte Walser auf den Schluss von Mozarts Die Entführung aus dem Serail Bezug nehmen, bei dem Konstanze dem Bassa Selim, den sie wegen Belmonte verlässt, gesteht, dass sie in allen Lebensmomenten an ihn denken werde: »Nie werd' ich im Genuß der Liebe / Vergessen, was der Dank gebeut«.

Lampersberg: Dornröschen [Klavierauszug], S. 43. 


\subsection{Epilog}

Es ist bisher nicht bekannt, wann und wo Lampersberg auf Robert Walser gestoßen ist, aber vermutlich war der bibliophile und vielseitig interessierte Komponist schon früh der Figur und den Texten von Walser begegnet. Wahrscheinlich hat auch Thomas Bernhard Walser im Tonhof kennengelernt. »Robert Walser liebe ich, seit ich ihn kenne und das ist drei Jahrzehnte lang «, schreibt Bernhard seinem Verleger Siegfried Unseld, der ihn 1978 zusammen mit vielen bedeutenden Schriftstellerinnen und Schriftstellern zur Hundertjahrfeier von Robert Walser nach Zürich eingeladen hat, ${ }^{29}$ also im Jahr der Uraufführung von Lampersbergs Dornröschen.

Leider ist dieses Werk seit der Uraufführung 1978 nicht mehr aufgeführt worden. Die internationale, jedoch fragwürdige Berühmtheit, die der Komponist durch Bernhards Holzfällen von 1984 erlangte, beeinträchtigte seine Karriere nachhaltig. Nicht einmal die späte Uraufführung seines bedeutendsten Werkes, das in Zusammenarbeit mit Bernhard entstanden war, konnte ihn rehabilitieren. Der damalige Intendant der Bonner Oper, Giancarlo del Monaco brachte 1995 als Regisseur Lampersbergs Die Rosen der Einöde in einer aufwendigen Produktion auf die Bühne, aber die gesamte Kritik betrachtete das Werk nur durch Bernhards Brille und stellte die musikalische Armut heraus. Der Opernkritiker Ulrich Schreiber formulierte es in der Frankfurter Rundschau vom 9. Dezember 1995 besonders scharf: »Wenn Thomas Bernhard Wien eine Talentzertrümmerungsanstalt nannte, so ist in Bonn deren Abdeckerei zu finden.« ${ }^{30}$ Die karge Ästhetik der 195oer-Jahre, in der Lampersberg komponierte, fand kein Gehör. Umgekehrt wurde immer wieder die >Musikalität< Bernhards gelobt. Diese Musikalität ist letztlich eine rhapsodische, die auf jenen Wiederholungs- und Affirmationsstrukturen beruht, die Bernhard gerade in Holzfällen besonders redundant verwendet. Genau diese persuadierende Form, diese den Hörer und Leser vereinnahmende Musikalität wollten die Musiker der 195oer-Jahre und insbesondere die Wiener Gruppe aber überwinden.

29 Bernhard/Unseld: Der Briefwechsel, S. 530. Der Zürcher Veranstaltung mit Gästen aus aller Welt blieb der im Voraus überall angekündigte Bernhard schließlich ohne Erklärung fern und bewirkte damit einen kleineren Skandal. Da er seinen Aufenthaltsort verschwieg, konnte auch der von der Stadt Zürich bereitgestellte Helikopter ihn nicht nach Zürich bringen.

Zit. nach Doppler: Holzfällen und der Tonhof, S. 210. 
Nach dem Skandal von Holzfällen legte Lampersberg seine Auffassung von $>$ Musikalität $<$ in zwei literarischen Arbeiten dar, in denen er sich auch über die Ménage-à-trois zwischen ihm, seiner Frau und Bernhard in den späten 195oerJahre äußerte: Einerseits publizierte er in kleiner Auflage 1987 die viersprachige dramatische Skizze Perturbation, andererseits veröffentlichte er zwei Jahre nach Bernhards Tod seine 1962 entstandene tagebuchartige Verarbeitung des Liebesverhältnisses als Diarium mitsamt rätselhaft dilettantisch wirkenden eigenen musikalischen Skizzen und Grafiken.

Die dramatischen Miniaturen von Perturbation imitieren den lakonischlapidaren Stil jener frühen Libretti, die Bernhard für Lampersberg von 1958 bis 196o geschrieben hat. Auf der Titelseite von Perturbation ist eine Fotografie von Bernhards in vier Stücke zerhacktem Holzfällen-Buch abgebildet. Perturbation ist in vier Sprachen verfasst und besteht aus drei Teilen mit je acht Gruppen von Mikro-Szenen, die aus wenigen Wörtern bestehen. Die drei Personen A, B und $C$ sprechen Englisch, Französisch und Deutsch, die Bühnenanweisungen stehen in Latein. In serieller Weise wird das identische Wortmaterial in neuen Konstellationen wiederholt.

Das Diarium im Gegenzug bearbeitet die Thematik der Dreierbeziehung in lyrischer Form. In drei Teilen wird aus der Perspektive von Anton, Anna und Joseph die Dreierbeziehung geschildert, auch hier unter Verwendung serieller Techniken und Satzpermutationen.

Permutation und Diarium sind beide als bibliophile Publikationen aufgemacht - auch dies eine Gegenposition zum die deutsche Literaturszene dominierenden Suhrkamp-Verlag - und bilden verzweifelte Versuche, Bernhards Sprachgewalt die kargen Mittel der Wiener Gruppe entgegenzuhalten. Beachtung fanden sie keine. Lampersberg ist selbst zu jenem Ausgestoßenen geworden, als den er am 11. Januar 1985 im ZEIT-Magazin noch selbstbewusst Thomas Bernhard bezeichnete:

Er ist ein total einsamer Mensch, fast wie ein Ausgestoßener Mensch. Seine Reaktion, alles, was er schreibt, ist die eines Ausgestoßenen. Ich bin das Gegenteil. Ich bin mittendrin. [...] Ich find' das wahnsinnig komisch, in Wirklichkeit muß ich eh nur lachen[.] $]^{31}$ 